\title{
Opiniones acerca de un objeto de aprendizaje en la asignatura de Pedagogía de la Actividad Física del Programa de Cultura Física, Deporte y Recreación
}

\author{
Luis Enrique Jiménez Garzón** \\ Recibido: diciembre 2 de 2016 • Evaluado: diciembre 15 de 2016
}

Aceptado: enero 5 de 2017

\section{Resumen}

Este escrito es un ensayo de reflexión crítica acerca de la creación de un objeto de aprendizaje (OA), en el programa virtual eXeLearning para el programa de Pedagogía de la Actividad Física de la Universidad Santo Tomás con sede en Bogotá (Colombia). Desde los aportes del Seminario temático I del Doctorado en Tecnología Educativa de la Universidad Mar de Cortés de México, se establece cuáles son las ventajas de crear una herramienta virtual que permita procesos de enseñanza y aprendizaje con mejores interacciones entre estudiantes y profesores. Finalmente, se plantean los aspectos más importantes de implementar un modelo virtual teniendo en cuenta experiencias anteriores en otros contextos educativos.

Palabras clave: objeto de aprendizaje, pedagogía, actividad física.

"* Licenciado en Educación Física de la Universidad de Cundinamarca. Especialista en Pedagogía del Entrenamiento Deportivo de la Universidad Pedagógica Nacional, y en Docencia Universitaria de la Universidad Cooperativa de Colombia. Magíster en Educación de la Universidad Santo Tomás y docente de la Facultad de Cultura Física, Deporte y Recreación de la misma institución. Correo electrónico: luisjimenezg@usantotomas.edu.co 


\section{Opinions about an object of learning in the subject of Pedagogy of the Physical Activity of the Physical Culture, Sports and Recreation Program}

\section{Abstract}

This essay is a critical reflection essay about the creation of a Learning Object (OA), in an exe-learning virtual program for the Pedagogy of Physical Activity program of the Santo Tomas University based in Bogota, Colombia. From the contributions of the thematic seminar I, of the doctorate in educational technologies of the University of Cortes de Mexico, a critical reflection is presented to raise opinions about what are the positive aspects of creating a virtual tool that allows processes of teaching and learning with better iterations between students and teachers. Finally, the most important aspects of implementing a virtual model are presented, considering previous experiences in other educational contexts.

Keywords: : Object of learning, Pedagogy, Physical activity. 


\section{Introducción}

En este escrito se expone parte del trabajo adelantado en el Seminario temático I del Doctorado en Tecnología Educativa de la Universidad Mar de Cortés de México. Se trata de una reflexión crítica sobre la tesis de grado que quiero desarrollar, denominada Opiniones acerca de un objeto de aprendizaje en la asignatura de Pedagogía de la Actividad Física. Para ese fin, se parte de materiales educativos y de investigación muy interesantes, como son el artículo científico "De los recursos de investigación a los objetos de aprendizaje: Modelo de Proceso y Experiencias de Virtualización" (Sierra et al., 2006) y los videos el "Desarrollo de Objetos de Aprendizaje" de Harvert Vanegas y "Objetos de Aprendizaje Reutilizables (Qué son)" de la Universidad Católica del Norte. En el ensayo expondré diversos conceptos teóricos sobre los objetos de aprendizaje (OA), a partir de los cuales planteo distintas opiniones con respecto a la idea de tesis de grado.

Este escrito pretende especificar las opiniones acerca de un objeto de aprendizaje en la asignatura de Pedagogía de la Actividad Física del Programa de Cultura Física, Deporte y Recreación en la Universidad Santo Tomás con sede en Bogotá (Colombia). Así mismo, busca responder a la siguiente pregunta: ¿cuáles son las opiniones acerca de un objeto de aprendizaje en la asignatura de Pedagogía de la Actividad Física?

En la Universidad Santo Tomás se encuentra el programa de Cultura Física, Deporte y Recreación, donde se forman profesionales en el contexto de la actividad física. Dentro de su currículo se encuentra la asignatura de Pedagogía de la Actividad Física, cuyos contenidos programáticos se pretenden virtualizar con un $\mathrm{OA}$, específicamente, la herramienta virtual $e X e$ Learning. Este ensayo da cuenta sobre las opiniones que tengo sobre los distintos conceptos necesarios para la construcción del OA en la asignatura de Pedagogía de la Actividad Física, apoyado desde experiencias educativas virtuales similares. La metodología de la tesis doctoral pertenece al campo de las ciencias sociales, de investigación cuantitativa y cualitativa, dentro de las fases de trabajo metodológico se plantearon diferentes fases como: 1) recolección de información referente al tema de objetos virtuales; 2 ) antecedentes de experiencias relacionadas con los objetos virtuales en la actividad física; 3) elaboración de un constructo teórico a partir de referentes conceptuales; 4) construcción de un cuestionario de recolección de datos acerca 
de las opiniones de docentes acerca de los objetos virtuales; 5$)$ resultados y análisis de los datos obtenidos y (6) planteamiento de conclusiones.

\section{Tecnologías de la información y la comunicación para la educación}

Es evidente el impacto de las herramientas virtuales en la sociedad y en especial en los distintos ámbitos educativos, pues han efectuado cambios en el rol del educador y del educando, en las metodologías y en el desarrollo de nuevas competencias de formación, como lo afirman Aguirre et al.: "actualmente se utilizan las tecnologías de información y comunicación para desarrollar y mejorar nuevas estrategias en el proceso de enseñanzaaprendizaje" (2009, p. 323). Los OA como herramientas virtuales aportan a la virtualización de los distintos contenidos y temáticas establecidas en cursos, diplomados, pregrados, posgrados y demás programas de una institución educativa.

\section{Generalidades sobre los objetos de aprendizaje}

Como primera medida, es importante definir qué es un OA: en el video "Objetos de Aprendizaje Reutilizables (Qué son)" de la Universidad Católica del Norte (2010), explican que para el Ministerio de Educación Nacional en Colombia y la Universidad de La Sabana un OA es una entidad digital autocontenible y reutilizable con un propósito educativo, constituido por unos componentes: contenidos, actividades y elementos de contextualización y que, además, debe tener una estructura externa llamada metadatos. Los OA también son elementos integrados e integradores del proceso de enseñanza-aprendizaje, que ofrecen a los estudiantes la posibilidad de mejorar su rendimiento y nivel de satisfacción (Vicerrectorado de Estudios y Convergencia Europea, 2006).

Desde estos conceptos sobre los OA se puede entender la importancia que cobran estas herramientas virtuales para la organización y virtualización de los distintos componentes del programa de actividad física, permitiendo al docente una mayor interacción con los estudiantes y el conocimiento. 
Entender estos conceptos es positivo y pertinente para la propuesta planteada en este ensayo.

Es válido para la construcción de un modelo virtual tener en cuenta las características de los OA (Vicerrectorado de Estudios y Convergencia Europea, 2006), que son:

- Formato digital: capacidad de actualización y/o modificación constante.

- Propósito pedagógico: asegurar un proceso de aprendizaje satisfactorio.

- Contenido interactivo: participación de cada individuo (profesor-alumno/as).

- Indivisible e independiente de otros OA: debe tener sentido en sí mismo.

- Reutilizable en contextos educativos distintos.

Partiendo de estas características como docente líder de esta propuesta, debo ser consciente de la importancia de los OA y buscar el máximo aprovechamiento de esta herramienta virtual para mejorar los ambientes teóricos y prácticos que se presentan en la asignatura de Pedagogía de la Actividad Física.

Dentro de los OA hay que tener en cuenta el concepto de reusabilidad, que puede darse en dos ámbitos (Universidad Católica del Norte, 2010):

- Desde lo educativo: existen muchos casos, uno de ellos puede ser cuando la institución quiere construir un curso, diplomado, programa, etc. Se debe contratar a un experto que indague acerca del material o recursos existentes (repositorio), seleccionando los temas.

- Desde lo tecnológico: la compatibilidad que tienen las plataformas para la construcción de objetos de aprendizaje.

Este concepto de reusabilidad "es difícil de medir ya que implica no sólo la evaluación de los contenidos por sí mismos, sino también un equilibrio entre su usabilidad en contextos específicos y la amplitud de los contextos de aprendizaje" (López et al., 2007, p. 1). Por otra parte, el hecho de que un $\mathrm{OA}$ sea reutilizable en contextos de aprendizaje demanda un diseño adecuado de sus contenidos y de sus metadatos, de forma tal que sean estables y perfectos para permitir procesos de selección. Así, la reusabilidad de 
los OA cobra importancia y rigurosidad para el proceso de construcción de un eXeLearning para la asignatura mencionada y es el docente quien desde su experiencia y conocimiento podrá seleccionar los temas adecuados para cada una de las unidades didácticas, actividades, metodologías y evaluaciones propuestas dentro del espacio académico, una característica que valoro de este tipo de herramientas virtuales.

Anteriormente, se aludió al término 'repositorio': este es el sitio centralizado y especializado de almacenamiento, donde para la organización y recuperación de los materiales se deben tener los respectivos metadatos (Universidad Católica del Norte, 2010). Además, se considera que un "repositorio es un concepto tan amplio que va desde sencillos sistemas de almacenamiento hasta complejos entornos que incorporan, además de los sistemas de almacenamiento, conjuntos de herramientas que ayudan al proceso de reutilización" (García, 2000, citado en López-Guzmán, 2005, p.41). El repositorio de los OA soporta la creación de la herramienta virtual, para el caso de la construcción del $\mathrm{OA}$ en el espacio académico contemplado en esta propuesta se usará programa eXeLearning. Considero que es indispensable tener una adecuada organización, acompañada de una rigurosidad en la selección del tema que se alojará dentro del repositorio, tarea fundamental que depende del docente y sus propósitos, sin apartarse del contexto educativo y las características de aprendizaje de los estudiantes.

El programa eXeLearning es una herramienta de código abierto (open source) que facilita la creación de contenidos educativos sin necesidad de ser un experto. Con este software se puede crear un árbol de contenidos básicos para facilitar la navegación, escribir textos y copiarlos desde otras aplicaciones, incluir imágenes, sonidos, vídeos y animaciones, preguntas de tipo test, de verdadero/falso, de espacios en blanco, exportar a SCORM e IMS para incluir los contenidos en plataformas como Moodle, exportar a ePub3, llevar contenidos al dispositivo móvil y reproducirlos sin necesidad de internet y otros beneficios más. Es por esta razón que considero que esta herramienta permitirá avanzar en la virtualización de los contenidos del programa, adecuar nuevas formas de enseñanza y aprendizaje en las clases, mejorará la interacción con los estudiantes y mantendrá un modelo de virtualización para la creación de futuros cursos afines a la pedagogía de la actividad física. 
Por otra parte, con la implementación de un OA mediante el software en mención, se da cumplimiento al Proyecto Educativo del Programa (PEP) propuesto por la Universidad Santo Tomás, que tiene como propósito el "mejoramiento permanente de sus niveles de calidad curricular. Este designio por la cualificación pedagógica implica el mejoramiento de los procesos docentes, investigativos y de proyección social para ofrecer servicios de calidad" (2010, p. 17). En consecuencia, como docente se deben tener las competencias adecuadas para los procesos de enseñanza y aprendizaje y dentro de estas competencias están el uso de las Tecnologías de la Información y la Comunicación (TIC), en el caso actual se trata de los OA, desde la utilización de la herramienta eXeLearning.

\section{Virtualización y prácticas educativas}

Dentro de los beneficios que trae la implementación de un modelo de virtualización como la herramienta eXeLearning se encuentra la mejora de las prácticas educativas. De Pablos y Jiménez definen que son unas buenas prácticas educativas en TIC "experiencias educativas y prácticas docentes que integran recursos digitales en software libre y redes de comunicación para crear nuevos contenidos y formas de organización escolar" (2007, p. 26). Además, las prácticas educativas
deben identificar principios adecuados para su realización, por ejemplo, promover las relaciones entre profesores y alumnos, desarrollar dinámi- cas de cooperación, aplicar técnicas activas para el aprendizaje, permitir la retroalimentación, enfatizar en el tiempo de dedicación a la tarea, co- municar altas expectativas y respetar la diversidad de formas de aprender (Chickering y Gamson, 1987, citados en De Pablos y Jiménez, 2007, p. 16).

Es por esta razón que resulta positivo pensar y entrar en acción con la construcción de un $\mathrm{OA}$ mediante eXeLearning, ya que beneficia a los estudiantes al brindarles opciones metodológicas de enseñanza y aprendizaje novedosas y acordes a sus expectativas con respecto al uso de las herramientas virtuales. 
Sierra et al. (2006) plantean un modelo de proceso de virtualización, cuyos tres principales componentes son:

- Productos y actividades: análisis de dominio (formulación del OA según los expertos); operacionalización (herramienta de creación y despliegue) y virtualización (el repositorio se llena con las virtualizaciones de los recursos).

- Secuenciación de las actividades: introduce iteraciones en la construcción de los repositorios; unas son correctivas (actualización y afinamiento de la herramienta de creación y despliegue) y otras evolutivas (nuevas características de los recursos).

- Participantes y responsabilidades: expertos y desarrolladores, en el análisis de dominio los desarrolladores formulan el modelo del OA y los expertos describen cómo se utiliza. En la operacionalización, la responsabilidad es para los desarrolladores, quienes deben construir la herramienta de creación y despliegue. Y en la virtualización, los expertos del dominio utilizan la herramienta de creación y despliegue del OA para rellenar el repositorio.

Otra experiencia en la implementación de un OA es la de Alonso et al. (2012), se trata de la Metodología para el Desarrollo de Objetos de Aprendizaje (MEDOA), que busca cumplir con los requisitos pedagógicos y tecnológicos adecuados. Tiene en cuenta las fases de planeación, análisis, diseño, implementación, validación, implantación y mantenimiento. Con respecto a estas experiencias podemos decir que son válidas desde que se haga una muy buena fase de diagnóstico, donde se proyecte el impacto que puede tener el $\mathrm{OA}$ en la población a intervenir. Además de esto, debe existir un control continuo sobre cada una de las fases de implementación evaluando la asertividad de los recursos que se van a utilizar para la creación del OA.

\section{Conclusiones}

- Un modelo de virtualización en el que se utilice la herramienta $e X e$ Learning para la asignatura de Pedagogía de la Actividad Física es pertinente desde que se tengan en cuenta las experiencias en otros contextos que han utilizado este tipo de OA. 
- Se busca crear este material virtual para que en un futuro otras asignaturas con el apoyo de sus tutores organicen nuevos OA.

- Se busca mediante este material virtual una herramienta que aporte a los procesos de aprendizaje activos y colaborativos, involucrando a profesores, estudiante y demás agentes que participen de los procesos de formación.

- La construcción del repositorio del OA debe tener en cuenta el material y recursos adecuados del espacio académico en mención y, además, debe cumplir con los propósitos de formación estipulados en las políticas de la Universidad Santo Tomás.

- Se busca el aprovechamiento del potencial del material del curso, brindando a los estudiantes un material virtual adecuado y que llene sus expectativas de aprendizaje.

- Los OA deben ser utilizados con fines pedagógicos y de investigación, con el fin de buscar nuevas experiencias en la pedagogía de la actividad física.

\section{Referencias}

Aguirre Andrade y Manasía N. (2009). Web 2.0 y Web semántica en los entornos virtuales de aprendizaje. Multiciencias, 9(3), 320-328.

Alonso, M., Castillo, I., Pozas, M., Curiel, M. y Trejo, L. (2012). Estandarizando los Objetos de Aprendizaje con MEDOA. Actas de la $7 \mathrm{ma}$ Conferencia Latinoamericana de Objetos de Aprendizaje. Guayaquil, Ecuador: EDITORIAL.

Católica del Norte (2010, marzo 4). Objetos de Aprendizaje Reutilizables (Qué son). Recuperado de https://www.youtube.com/watch?v=-Bmk3ATK67s\&t=3s.

De Pablos Pons, J. y Jiménez Cortés, R. (2007). Buenas prácticas con TIC apoyadas en las Políticas Educativas: claves conceptuales y derivaciones para la formación en competencias ECTS. Revista Latinoamericana de Tecnología Educativa, 6(2), 15-28.

Facultad de Cultura Física, Deporte y Recreación (2012). Proyecto Educativo del Programa. Bogotá, Colombia: USTA.

López-Guzmán, C. (2005). Los Repositorios de Objetos de Aprendizaje como soporte a un entorno e-learning (tesis de doctorado). Universidad de Salamanca, Salamanca. 
López, M.G., Maestre Escalante, A.J., Sánchez Alonso, S. Reusabilidad de los Objetos de Aprendizaje almacenados en Repositorios de Libre Acceso. En actas de SPDECE 2007, IV Simposio Pluridisciplinar sobre Diseño, Evaluación y Desarrollo de Contenidos Educativos Reutilizables. Bilbao, España. http:// spdece07.ehu.es/actas/Lopez.pdf . Accedido en septiembre de 2007.

Sierra, J. L., Fernández-Valmayor, A., Guinea, M. y Hernanz, H. (2006). From Research Resources to Learning Objects: Process Model and Virtualization Experiences. Educational Technology \& Society, 9(3), 56-68.

Universidad Católica del Norte (2010). Objetos de Aprendizaje Reutilizables (Qué son). Recuperado de https://www.youtube.com/watch? $v=-B m k 3 A T K 67 \mathrm{~s} \& \mathrm{t}=5 \mathrm{~s}$

Vanegas, Harvert (2013, noviembre 5). Desarrollo de Objetos de Aprendizaje. Recuperado de https://www.youtube.com/watch?v=0TKOhu3GNuA

Vicerrectorado de Estudios y Convergencia Europea. "Plan de Acciones para la Convergencia Europea (PACE)”. Universidad Politécnica de Valencia. 2006. 